

\title{
Meromorphic continuation for the zeta function of a Dwork hypersurface
}

\author{
Thomas Barnet-Lamb
}

\begin{abstract}
We consider the one-parameter family of hypersurfaces in $\mathbb{P}^{5}$ over $\mathbb{Q}$ with projective equation $\left(X_{1}^{5}+X_{2}^{5}+X_{3}^{5}+X_{4}^{5}+X_{5}^{5}\right)=5 t X_{1} X_{2} \ldots X_{5}$, proving that the Galois representations attached to their cohomologies are potentially automorphic, and hence that the zeta function of the family has meromorphic continuation to the whole complex plane.
\end{abstract}

\section{Introduction}

Harris, Shepherd-Barron, and Taylor have proved in [Harris et al. 2010] a potential modularity theorem, showing that certain Galois representations become automorphic after a sufficiently large totally real base change. In their argument, a key role is played by certain families of hypersurfaces, called Dwork families - in particular, by the part of the cohomology of the family which is invariant under a certain group action. (We will write $\mathfrak{F}$ for the motive given by this part of the cohomology.) The importance of $\mathfrak{F}$ to their argument is reflected in the statement of the theorem they prove: in order to prove an $l$-adic Galois representation $r$ is potentially modular using their theorem, one requires, among other conditions, that the restriction of the residual representation of $r$ to inertia at primes above $l$ be isomorphic to the restriction of the residual representation of some element of the family $\mathfrak{F}$.

They give two applications in their paper. On the one hand, through considerable ingenuity (and the fact that the Dwork family includes the Fermat hypersurface, whose cohomology restricted to inertia is easy to analyze) they are able to deduce that the odd symmetric powers of the cohomology of an elliptic curve over $\mathbb{Q}$ are modular, and (through further ingenuity) to deduce the Sato-Tate conjecture. On the other hand, the form of the condition on the inertial representation makes it very inviting to apply their modularity theorem to $\mathfrak{F}$ itself. It turns out to be fairly simple

The author was partially supported by NSF grant DMS-0600716 and by a Jean E. de Valpine Fellowship.

MSC2000: primary 11G40; secondary 11R39, 11F23.

Keywords: Dwork hypersurface, potential automorphy, zeta function. 
to show that the other conditions of the potential modularity theorem are satisfied, and one can deduce the modularity of $\mathfrak{F}$ and hence the meromorphic continuation and functional equation of the zeta function of this part of the cohomology of the Dwork family.

A very natural question presents itself: is it possible to gain enough control of the other parts of the cohomology of the Dwork family that one can prove meromorphic continuation for the whole zeta function? In this paper, I answer this question in the affirmative for $N=5$, and also make some remarks on why further generalization of these methods is likely to be hard absent very significant advances in the technology of lifting theorems. In the analysis here, a key role is played by [Katz 2009], which describes the relative cohomology sheaf of the Dwork family over the base, and its decomposition under the group action alluded to above, in great detail.

Note. Since this paper was written, the technology of potential automorphy has advanced somewhat; in particular, [Barnet-Lamb et al. 2010] proves certain rather general potential automorphy theorems for regular, crystalline, self-dual representations of the Galois group of totally real and CM fields. Using those results, the argument for Proposition 5-which is perhaps rather involved at present - could be replaced with an appeal to the theorems for compatible systems proved in the new manuscript. The remainder of the present paper, in particular the analysis of the pieces of the cohomology in Section 3, does not seem to be able to be simplified, even with these new results.

\section{Dwork families}

Note. My notation for Dwork families broadly follows [Katz 2009], with $N$ in place of $n$, except that Katz works throughout with sheaves with coefficients in $\overline{\mathbb{Q}}_{l}$, whereas we will need the flexibility gained by working initially with $\mathbb{Q}_{l}$ coefficients, and extending to $\overline{\mathbb{Q}}_{l}$ only as necessary to apply Katz's results. Our notation is not directly comparable with the notation of [Harris et al. 2010].

Let $N$ be a positive integer. Fix a base ring

$$
R_{0}=\mathbb{Z}\left[1 / N, \mu_{N}\right]
$$

where $\mu_{N}$ denotes the $N$-th roots of unity. For most of this paper, we work over $\mathbb{Q}\left(\mu_{5}\right)$ and all Galois representations are representations of subgroups of $G_{\mathbb{Q}\left(\mu_{5}\right)}$. We will eventually return to working over $\mathbb{Q}$, but when we do so, this will be made explicit. We consider the scheme

$$
Y \subset \mathbb{P}^{N-1} \times \mathbb{P}^{1}
$$

over $R_{0}$ defined by the equations

$$
\mu\left(X_{1}^{N}+X_{2}^{N}+\cdots+X_{N}^{N}\right)=N \lambda X_{1} X_{2} \ldots X_{N},
$$


using $\left(X_{1}: \cdots: X_{N}\right)$ and $(\mu: \lambda)$ as coordinates on $\mathbb{P}^{N-1}$ and $\mathbb{P}^{1}$ respectively. We consider $Y$ as a family of schemes over $\mathbb{P}^{1}$ by projection to the second factor. We will label points on this $\mathbb{P}^{1}$ using the affine coordinate $t=\lambda / \mu$, and will write $Y_{t}$ for the fiber of $Y$ above $t$. From now on (apart from some remarks in the conclusion) we will be concerned exclusively with the case $N=5$.

The family $Y$ is smooth over the open set $U=\operatorname{Spec} R_{0}\left[1 /\left(t^{5}-1\right)\right]$ away from the roots of unity. We are interested in the sheaf of relative cohomology of the family $Y$ above the set $U$. Let $l$ be a prime number which splits in $\mathbb{Q}\left(\mu_{5}\right) .{ }^{1}$ Let $T_{0}=U[1 / l]$, and form lisse sheaves

$$
\begin{gathered}
\mathscr{F}_{l}^{i}:=R^{i} \pi_{*} \mathbb{Q}_{l}, \\
\mathscr{F}^{i}[l]:=R^{i} \pi_{*} \mathbb{Z} / l \mathbb{Z}
\end{gathered}
$$

on $T_{0}$. (We remark that Katz's $\mathscr{F}_{l}^{i}$ would be $\mathscr{F}_{l}^{i} \otimes \overline{\mathbb{Q}}_{l}$ in our notation, since he works with algebraically closed coefficients throughout.) As a family of hypersurfaces, much of the cohomology of $Y_{\lambda}$ is controlled by the hard Lefschetz theorem: for $i \neq N-2=3$, we have

$$
\mathscr{F}_{l}^{i}= \begin{cases}0 & (i<0), \\ 0 & (i>6), \\ 0 & (i \text { odd }, 0 \leq i \leq 6), \\ \mathbb{Q}_{l}(-j) & (i=2 j \text { even, } 0 \leq i \leq 6, i \neq 3) .\end{cases}
$$

The contribution to the zeta function from the characters $\mathbb{Q}_{l}(-j)$ is of course well understood. Thus in order to prove the functional equation for the zeta function of the whole variety, it suffices to control the zeta function of $\mathscr{F}_{l}^{3}$. We will refer to the sheaf $\mathscr{F}_{l}^{3}$ as $\operatorname{Prim}_{l}$ from now on. As discussed in the introduction, there is a natural group action on $\operatorname{Prim}_{l}$, allowing us to break down the cohomology into simpler pieces. Let us now introduce this group action. We will write $\Gamma$ for $\left(\mu_{5}\right)^{5}$, the 5 -fold product of the group of roots of unity, and $\Gamma_{W}$ for the subgroup of elements $\left(\zeta_{1}, \ldots \zeta_{5}\right)$ with $\prod_{i=1}^{5} \zeta_{i}=1$. $\Gamma_{W}$ acts on $Y$ with $\left(\zeta_{1}, \ldots \zeta_{5}\right)$ acting via

$$
\left(\left(X_{1}: \cdots: X_{5}\right), t\right) \mapsto\left(\left(\zeta_{1} X_{1}: \cdots: \zeta_{5} X_{5}\right), t\right) .
$$

The image of $\mu_{5}$ embedded diagonally in $\Gamma$ lies in $\Gamma_{W}$ and acts trivially under this action. We will write $\Delta$ for this image.

Harris et al. [2010] focus their attention on the invariants under this group action, a sheaf they refer to as $V$. They prove the following theorem:

\footnotetext{
${ }^{1}$ I believe that this assumption could be dispensed with. However, we will only ever need the theory we are about to develop for one particular choice of $l$, and we will always be able to make this choice such that $l$ splits in $\mathbb{Q}\left(\mu_{5}\right)$. Therefore, I have chosen to make this assumption, since it simplifies the argument. In particular, it means that the sheaves $\operatorname{Prim}_{l}$ which we define later will have coefficient ring $\mathbb{Q}_{l}$, rather than an extension field.
} 
Theorem 1 [Harris et al. 2010, Theorem 4.4]. Suppose that $t \in \mathbb{Q}-\mathbb{Z}[1 / 5]$. Then the function $L\left(V_{t}, s\right)$ is defined and has meromorphic continuation to the whole complex plane, satisfying the functional equation

$$
L(V, s)=\epsilon(V, s) L(V, 4-s) .
$$

As I have said, our aim in this paper is to analyze the remaining parts of the cohomology and so establish the functional equation for the zeta function of the variety as a whole. As a first step to doing so, let us consider what other parts there actually are.

\section{The pieces of the cohomology}

The character group of $\Gamma$ is $(\mathbb{Z} / 5 \mathbb{Z})^{5}$; that of $\Gamma_{W}$ is $(\mathbb{Z} / 5 \mathbb{Z})^{5} /\langle W\rangle$ where we write $W$ for the element $(1,1, \ldots, 1)$; and that of $\Gamma_{W} / \Delta$ is $(\mathbb{Z} / 5 \mathbb{Z})_{0}^{5} /\langle W\rangle$ where we write $(\mathbb{Z} / 5 \mathbb{Z})_{0}^{5}$ for $\left\{\left(v_{1}, \ldots, v_{5}\right) \in(\mathbb{Z} / 5 \mathbb{Z})^{5} \mid \sum_{i} v_{i}=0\right\}$. Thus the eigensheaves of $\operatorname{Prim}_{l}$ under the action described in Section 2 are labeled by elements $v$ of $(\mathbb{Z} / 5 \mathbb{Z})_{0}^{5} /\langle W\rangle$ : we may write such an element as $\left(v_{1}, \ldots, v_{5}\right) \bmod W$ with the $v_{i}$ elements of $\mathbb{Z} / 5 \mathbb{Z}$; it will often be convenient to abbreviate this to $\left[\left(v_{1}, \ldots, v_{5}\right)\right]$. Note that our assumption that $\mu_{5} \in \mathbb{Q}_{l}$ is critical here in ensuring that the decomposition into eigensheaves is indeed defined with $\mathbb{Q}_{l}$ coefficients (and not with coefficients in some extension field). Note also that the labeling is not canonical, but depends on a choice of an identification of the copies of $\mu_{5}$ in $\mathbb{Q}_{l}$ and in $R_{0}$ : equivalently, it depends on a choice of embedding $R_{0} \hookrightarrow \mathbb{Q}_{l}$. Having made such a choice, we shall write $\operatorname{Prim}_{l,\left[\left(v_{1}, \ldots, v_{5}\right)\right]}$ for the piece of $\operatorname{Prim}_{l}$ where $\Gamma_{W} / \Delta$ acts via $\left[\left(v_{1}, \ldots, v_{5}\right)\right]$. (Thus, for instance, $V_{l}=\operatorname{Prim}_{l,[(0,0,0,0,0)]}$.) Again, we remark that Katz's $\operatorname{Prim}_{l, v}$ would correspond to our $\operatorname{Prim}_{l, v} \otimes \overline{\mathbb{Q}}_{l}$.

The obvious action of $S_{5}$ on $(\mathbb{Z} / 5 \mathbb{Z})^{5}$ preserves $(\mathbb{Z} / 5 \mathbb{Z})_{0}^{5}$ and $W$, and hence induces an action of $S_{5}$ on $(\mathbb{Z} / 5 \mathbb{Z})_{0}^{5} /\langle W\rangle$. Note that, if we permute the $\left(v_{i}\right)$ in this manner, the resulting sheaf $\operatorname{Prim}_{l, v}$ is isomorphic to the original (the isomorphism being induced from the map on $Y$ which permutes the $X_{i}$ according to the same permutation). Thus to show that all the sheaves $\operatorname{Prim}_{l, v}$ are automorphic it will suffice to consider a set of $v$ representing all the orbits of $(\mathbb{Z} / 5 \mathbb{Z})_{0}^{5} /\langle W\rangle$ under $S_{5}$.

Proposition 2. All orbits of $(\mathbb{Z} / 5 \mathbb{Z})_{0}^{5} /\langle W\rangle$ under $S_{5}$ are represented in the list

$$
\begin{aligned}
& {[(0,1,2,3,4)],[(0,0,1,1,3)],[(0,0,1,2,2)],[(0,0,2,4,4)],} \\
& {[(0,0,3,3,4)],[(0,0,0,1,4)],[(0,0,0,2,3)],[(0,0,0,0,0)] .}
\end{aligned}
$$

Thus, if $\operatorname{Prim}_{l, v}$ is automorphic for each of these $v \in(\mathbb{Z} / 5 \mathbb{Z})_{0}^{5}$, it is automorphic for all $v$.

Proof. We start with an arbitrary element $v$ of $(\mathbb{Z} / 5 \mathbb{Z})_{0}^{5} /\langle W\rangle$, and pick a representative $\left(v_{1}, \ldots, v_{n}\right) \in(\mathbb{Z} / 5 \mathbb{Z})_{0}^{5}$. By changing the representative of the congruence 
class $\bmod W$, we may ensure that in the list $\left(v_{1}, \ldots, v_{5}\right)$, the 0 occurs at least as often as any other element of $\mathbb{Z} / 5 \mathbb{Z}$. Then, applying an appropriate permutation to $v$ (and hence to the $v_{i}$ ), we may ensure that the $v_{i}$ increase. (We order congruence classes mod 5 according to the order of their unique representatives in $\{0, \ldots, 4\}$.)

Since 0 occurs at least as often as anything else, there must be at least one zero at the beginning of the list $\left(v_{1}, \ldots, v_{5}\right)$. We split into several cases according to the number of zeroes there. Clearly, if there is 1 zero then $v=[(0,1,2,3,4)]$; if there are 4 or more zeros then $v=[(0,0,0,0,0)]$; and if there are 3 then the two remaining $v_{i}$ are 1 and 4 or 2 and 3 .

If there are 2 zeroes, then we split into cases according to the value of $v_{3}$. If, for instance, $v_{3}=1$, then $v_{4}+v_{5}=4$, so $v_{4}, v_{5}$ must be $\{1,3\},\{2,2\}$, or $\{0,4\}$, the last being impossible since the $v_{i}$ must increase. The other cases are similar.

Proposition 3. Assume we have chosen an arbitrary embedding of $R_{0}$ into $\overline{\mathbb{Q}}_{l}$. For each $v$ in the following table, the dimension and Hodge-Tate numbers of $\operatorname{Prim}_{l, v}$ are as given:

$\begin{array}{ccc}v & \operatorname{dim}_{\operatorname{Prim}} \operatorname{HT}_{l, v} & \left.\mathrm{HT} \operatorname{Prim}_{l, v}\right) \\ {[(0,1,2,3,4)]} & 0 & \{\} \\ {[(0,0,1,1,3)]} & 2 & \{1,2\} \\ {[(0,0,1,2,2)]} & 2 & \{1,2\} \\ {[(0,0,2,4,4)]} & 2 & \{1,2\} \\ {[(0,0,3,3,4)]} & 2 & \{1,2\} \\ {[(0,0,0,1,4)]} & 2 & \{1,2\} \\ {[(0,0,0,2,3)]} & 2 & \{1,2\}\end{array}$

In particular, $\operatorname{Prim}_{l,[(0,1,2,3,4)]}$ is zero-dimensional, and although the Hodge-Tate numbers depend in principle on the choice of embedding of $R_{0}$ into $\mathbb{Q}_{l}$, in practice they are independent of this choice.

Proof. Recall that at the beginning of this section we chose a particular embedding $R_{0} \rightarrow \mathbb{Q}_{l}$ in order to label the pieces of the cohomology. (We remark that since $R_{0}=\mathbb{Z}\left[1 / N, \mu_{n}\right]$ and we have a running assumption that $\mu_{N} \subset \mathbb{Q}_{l}$, this is the same thing as choosing an embedding $R_{0} \hookrightarrow \overline{\mathbb{Q}}_{l}$.) Katz makes a corresponding choice in [Katz 2009, §1], and the Hodge-Tate numbers at this particular embedding (as well as the dimension, which does not depend on the choice of an embedding) may then be calculated by applying the procedure described in [ibid., Lemma 3.1]. (We will investigate what happens for Hodge-Tate numbers at the other embeddings later.) More precisely, Katz's procedure computes the Hodge-Tate numbers for his sheaf $\operatorname{Prim}_{l, v}$, which is our $\operatorname{Prim}_{l, v} \otimes_{\mathbb{Q}_{l}} \overline{\mathbb{Q}}_{l}$, but of course the Hodge-Tate numbers of $\operatorname{Prim}_{l, v}$ and $\operatorname{Prim}_{l, v} \otimes_{\mathbb{Q}_{l}} \overline{\mathbb{Q}}_{l}$ are the same. 
As an example, we will compute the dimension and Hodge-Tate numbers for $v=[(0,0,1,1,3)]$. We are asked to consider the coset of elements of $(\mathbb{Z} / 5 \mathbb{Z})_{0}^{5}$ representing $v=[(0,0,1,1,3)]$, that is to say the particular embedding $R_{0} \rightarrow \mathbb{Q}_{l}$ which was chosen arbitrarily at the beginning of this section (or in [ibid., §1]) and used to label the pieces of the cohomology

$$
\{(0,0,1,1,3),(1,1,2,2,4),(2,2,3,3,0),(3,3,4,4,1),(4,4,0,0,2)\} .
$$

The lemma then tells us that the dimension of $\operatorname{Prim}_{l}$ can be computed as the number of elements of this set which are totally nonzero; that is, contain no 0's. There are two of these. Then, the Hodge-Tate numbers are computed by taking the degrees of the totally nonzero representatives above, where the degree of an element $\left(v_{1}, \ldots, v_{n}\right) \in(\mathbb{Z} / 5 \mathbb{Z})_{0}^{5}$ is $\sum_{i} \tilde{v}_{i}$, and where (in turn) for each $i, \tilde{v}_{i}$ is the integer representing $v_{i}$ in the range 0 to 4 . Then the multiset of these degrees is the multiset of Hodge-Tate numbers, with each element increased by 1 . In our case, the HT numbers are therefore $\{(1+1+2+2+4) / 5-1,(3+3+4+4+1) / 5-1\}=\{1,2\}$.

For the other $v$ 's, the totally nonzero representatives are as follows:

$$
\begin{array}{cc}
v & \text { Totally nonzero representatives } \\
{[(0,0,1,2,2)]} & \{(1,1,2,3,3),(2,2,3,4,4)\} \\
{[(0,0,2,4,4)]} & \{(2,2,4,1,1),(4,4,1,3,3)\} \\
{[(0,0,3,3,4)]} & \{(3,3,1,1,2),(4,4,2,2,3)\} \\
{[(0,0,0,1,4)]} & \{(2,2,2,3,1),(3,3,3,4,2)\} \\
{[(0,0,0,2,3)]} & \{(1,1,1,3,4),(4,4,4,1,2)\}
\end{array}
$$

The result, for the Hodge-Tate numbers at our chosen embedding, follows.

Now, when we change our choice of embedding, the effect is to relabel the various pieces of the cohomology, by multiplying their labels $v$ by an element of $(\mathbb{Z} / 5 \mathbb{Z})^{\times}$. It is easy to see from the table that such relabeling sends an eigenspace to another eigenspace where the calculated Hodge-Tate numbers from the algorithm are the same. Hence we are done.

\section{Controlling the $L$ functions}

We will now try to control the $L$ functions of the two-dimensional pieces we have singled out.

Lemma 4. Let $v$ be taken from the table in Proposition 3. There is a constant $D$ such that if $M$ is an integer divisible only by primes $p>D$ and if $t \in U$ then the map

$$
\pi_{1}(U, t) \rightarrow \operatorname{SL}\left(\operatorname{Prim}[M]_{v, t}\right)
$$


is surjective. (Here $\mathrm{SL}\left(\operatorname{Prim}[M]_{t}\right.$ ) denotes the group of automorphisms of the twodimensional module $\operatorname{Prim}[M]_{t}$ with determinant 1.$)$

Proof. The monodromy of $\operatorname{Prim}_{l} \otimes \overline{\mathbb{Q}}_{l}$ is Zariski dense in $\mathrm{SL}_{2}\left(\operatorname{Prim}_{l}\right)$, using [Katz 2009, Lemma 10.3], and remembering that $\mathrm{Sp}_{2}=\mathrm{SL}_{2}$. The same is then seen to hold for $\operatorname{Prim}_{l}$. We can then deduce the result using [Matthews et al. 1984, Theorem 7.5 and Lemma 8.4] or [Nori 1987, Theorem 5.1].

We now proceed to analyze the two-dimensional pieces. We shall write Prim $*, v$ to mean the motive whose $l$-adic realizations are the $\operatorname{Prim}_{l, v}$ as $l$ varies.

Proposition 5. For each $v$ in the table of Proposition 3 with Prim $_{*, v}$ two-dimensional, and for each $t \in \mathbb{Q}-\mathbb{Z}[1 / 10]$, the function $L\left(\operatorname{Prim}_{*, v, t}, s\right)$ is defined and has meromorphic continuation to the whole complex plane, satisfying the functional equation

$$
L\left(\operatorname{Prim}_{*, v, t}, s\right)=\epsilon\left(\operatorname{Prim}_{*, v, t}, s\right) L\left(\operatorname{Prim}_{*, v^{*}, t}, 4-s\right),
$$

where we write $v^{*}$ for $\{5-k \mid k \in v\}$.

Before proving this, let us briefly remind ourselves of the significance of the words "is defined" in the statement of the theorem. The point is that, for each prime $p$, we wish to construct a local $L$ factor $L_{p}$, and we do so by looking at our motive's $l$-adic cohomology $\operatorname{Prim}_{l, v, t}$ for some $l \neq p$. Given an embedding $\overline{\mathbb{Q}} \hookrightarrow \overline{\mathbb{Q}}_{l}$, we can associate a Weil-Deligne representation $\mathrm{WD}\left(\left.\operatorname{Prim}{ }_{l, v, t}\right|_{\mathrm{Gal}\left(\overline{\mathbb{Q}}_{p} / \mathbb{Q}_{p}\right)}\right)^{\mathrm{F}-\mathrm{ss}}$ to this $l$ adic cohomology at $p$, and to this, in turn, we can associate an $L$ factor. To get an unambiguous $L$ factor, we must insist that the Weil-Deligne representation (and hence the $L$ factor) do not depend on the choices we made: that is, the choice of $l$ and of an embedding $\overline{\mathbb{Q}} \hookrightarrow \overline{\mathbb{Q}}_{l}$. Thus the statement " $L\left(\operatorname{Prim}_{*, v, t}, s\right)$ is defined" is saying that for every $p$, the local Weil-Deligne representation at $p$ constructed in this way is independent of these choices.

Proof of Proposition 5. Our argument draws heavily on Theorem 3.3 of [Harris et al. 2010]. We first choose $q$ to be a rational prime dividing the denominator of $t$, so that $v_{q}(t)<0$ and $q \nmid 10$.

Step 1. The goal of this step is to choose certain primes $l$ and $l^{\prime}$ which will be instrumental to the argument. In order to be in a position to do this we must first analyze the Zariski closure of the image of $\operatorname{Gal}\left(\overline{\mathbb{Q}} / \mathbb{Q}\left(\mu_{5}\right)\right)$ in the group $\operatorname{GL}\left(\operatorname{Prim}_{l, v, t}\right)$ of automorphisms of the $\mathbb{Q}_{l}$ vector space $\operatorname{Prim}_{l, v, t}$. We will write $G_{l}$ for this image and $G_{l}^{0}$ for the connected component of the identity in it.

By [Katz 2009, Lemma 10.1] the local monodromy of $\operatorname{Prim}_{l, v, t} \otimes \overline{\mathbb{Q}}_{l}$ at $\infty$ is unipotent with a single Jordan block. (Condition 4 of the equivalent conditions in that lemma may be verified by direct inspection of each case in Section 3.) We immediately deduce the same for Prim $\operatorname{Piv}_{l, t}$ itself. By the argument used to establish [Harris et al. 2010, Lemma 1.15], and recalling that $v_{q}(t)<0$, we conclude 
that inertia at $q$ acts via a maximal unipotent. Thus $G_{0}^{l}$ contains such a maximal unipotent, and hence, by [Scholl 2006, Proposition 3], acts irreducibly.

Moreover, the determinant map to $\mathbb{G}_{m}$ is dominating. To see this, note that Poincaré duality furnishes us with a perfect pairing between $\operatorname{Prim}_{l, v, t}$ and $\operatorname{Prim}_{l, v^{*}, t}$ towards $\mathbb{Q}(-3)$, and that $\operatorname{Prim}_{l, v^{*}, t}$ is the complex conjugate of $\operatorname{Prim}_{l, v, t}$. Thus we have

$$
\operatorname{Prim}_{l, v, t}^{c}=\operatorname{Prim}_{l, v, t}^{\vee} \epsilon_{l}^{-3},
$$

which tells us in turn that $\left(\operatorname{det}_{\operatorname{Prim}}^{l, v, t}\right)\left(\operatorname{det} \operatorname{Prim}_{l, v, t}\right)^{c}=\epsilon_{l}^{-6}$, which would be impossible if the determinant character did not dominate $\mathbb{G}_{m}$.

Thus by [Katz 1988, Theorem 9.10], we may conclude that $G_{l}^{0}$ is $\mathrm{GL}_{2}$. The main theorem of [Larsen 1995] then tells us that the set of primes $l$ for which we fail to have

$$
\mathrm{PSL}_{2}\left(\operatorname{Prim}[l]_{v, t}\right) \subset \Gamma_{l} \subset \mathrm{PGL}_{2}\left(\operatorname{Prim}[l]_{v, t}\right)
$$

has Dirichlet density 0.

By mimicking the argument for Proposition 3.4.2 of [Barnet-Lamb 2008], we can construct a field $F^{*}(v)$ such that if a prime $l$ splits in $F^{*}(v)$, the determinant of the natural polarization on Prim $[l]_{v}$ coming from Poincaré duality will be a square. (We also take from this paper two notations which we will use a few lines below: the field $F^{*}(2,10)$ and the integer $C(2,10)$, defined respectively in Proposition 3.4.2 and Corollary 2.1.2 there.) Now, since the set of primes for which (4) holds had Dirichlet density 1, the set of primes for which (4) holds and for which $l$ splits in $\mathbb{Q}\left(\mu_{10}\right)$, in $F^{*}(v)$ and in $F^{*}(2,10)$ has positive density. We may therefore choose $l$ to be such a prime, and in addition insist that

$$
v_{l}\left(t^{5}-1\right)=0
$$

and that $l$ be greater than $n, C(2,10)$ and $D$ (see Lemma 4 for the latter).

We choose $l^{\prime}$ to be a distinct rational prime enjoying the same list of properties. Note that (4) will ensure that the image of $\operatorname{Gal}\left(\overline{\mathbb{Q}} / \mathbb{Q}\left(\zeta_{l}\right)\right)$ in $\operatorname{GL}\left(\operatorname{Prim}[l]_{t}\right)$ is big, via (say) [Clozel et al. 2008, Lemma 2.5.5], and the simplicity of $\mathrm{PSL}_{2}\left(\mathbb{F}_{l}\right)$ will ensure that $\zeta_{l} \notin \overline{\mathbb{Q}}^{\mathrm{ker} \operatorname{Prim}\left[l_{t}\right.}$.

Step 2. Our next step in the proof is to establish that there exists a CM field $F_{1} / \mathbb{Q}\left(\mu_{10}\right)$ and a $t^{\prime} \in T\left(F_{1}\right)$ such that we have

$$
\begin{aligned}
\operatorname{Prim}[l]_{v, t^{\prime}} & \equiv \operatorname{Prim}[l]_{v, t}, \\
\left.\operatorname{Prim}\left[l^{\prime}\right]_{v, t^{\prime}}\right|_{I_{F_{1, \mathfrak{w}}}} & \equiv \epsilon_{l}^{-1} \oplus \epsilon_{l}^{-2}, \\
v_{\mathfrak{q}}\left(t^{\prime}\right) & <0 \quad \text { if } \mathfrak{q} \mid q, \\
v_{\mathfrak{w}}\left(\left(t^{\prime}\right)^{5}-1\right) & =0 \quad \text { if } \mathfrak{w} \mid l l^{\prime} .
\end{aligned}
$$

First, pick a point $t^{\prime \prime} \in \mathbb{Q}\left(\mu_{10}\right)^{+}$satisfying the following conditions: 
- If $\mathfrak{w} \mid l l^{\prime}$ then $w\left(\left(t^{\prime \prime}\right)^{5}-1\right)=0$.

- If $\mathfrak{w} \mid l^{\prime}$ then Prim $\left.\left[l^{\prime}\right]_{t^{\prime \prime}}\right|_{I_{\mathfrak{w}}} \equiv \epsilon_{l}^{-1} \oplus \epsilon_{l}^{-2}$.

- $\operatorname{Gal}\left(\overline{\mathbb{Q}} / \mathbb{Q}\left(\mu_{10}\right)^{+}\right) \rightarrow \operatorname{SL}\left(\operatorname{Prim}\left[l^{\prime}\right]_{t^{\prime \prime}}\right)$ is surjective.

As in [Harris et al. 2010], the existence of such a $t^{\prime \prime}$ relies on the form of Hilbert irreducibility with weak approximation; see [Ekedahl 1990]. We may achieve the second condition by taking $t^{\prime \prime}$ to be $l^{\prime}$-adically close to zero, since Prim $\left.\left[l^{\prime}\right]_{0}\right|_{I_{w}}$ is $\epsilon_{l}^{-1} \oplus \epsilon_{l}^{-2}$. (This last is because we know Prim $\left.\left[l^{\prime}\right]_{0}\right|_{I_{w}}$ to be a direct sum of characters, as in [Deligne et al. 1982], and we know from Proposition 3 that they are crystalline with Hodge-Tate numbers 1,2.)

We introduce the character $\phi_{l}$ as is done in Section 3.2 of [Barnet-Lamb 2008]. All references through the end of this step are to that paper. ${ }^{2}$ We will also consider the $l^{\prime}$-adic version of $\phi$, as well as the $\bmod M:=l l^{\prime}$ version; we will abuse notation by writing $\phi$ for all of these.

Now, we follow the argument of the proof of Proposition 3.4.1, with this setup: $l_{1}=l, l_{2}=l^{\prime}, \bar{\rho}_{1}=\operatorname{Prim}[l]_{v, t} \otimes \phi^{-1}, \bar{\rho}_{2}=\operatorname{Prim}\left[l^{\prime}\right]_{v, t^{\prime \prime}} \otimes \phi^{-1}$, the $\mathfrak{q}_{j}$ are the primes above $q$, and $N=5$. Details follow:

The first part of the argument works in exactly the same way: we set $M:=l l^{\prime}$, introduce a $\bmod M$ character $\phi_{l}$ and a $\bmod M$ representation $\bar{\rho}_{\mathbb{Z} / M \mathbb{Z}}$, and note that $\operatorname{Prim}[M]$ and $\bar{\rho}_{\mathbb{Z} / M \mathbb{Z}}$ become isomorphic once we disregard the Galois action and keep only the modules with a pairing, using the assumption that $l$ splits in $F^{*}(v)$.

We next study the determinant of Prim. From the fact that $\psi_{1}$ maps into the image of geometric monodromy, we can deduce that it is trivial, since we saw above that geometric monodromy was trivial. Thus we $\operatorname{deduce}$ that $\operatorname{det}\left(\operatorname{Prim}[l]_{s} \otimes \phi^{-1}\right)$ is independent of $s$ - in fact, from an argument analogous to that establishing Lemma 3.2.1, we know that $\operatorname{det}\left(\operatorname{Prim}[l]_{s} \otimes \phi^{-1}\right)=\epsilon_{l}^{-1}$. Similarly, $\operatorname{det}\left(\operatorname{Prim}[l]_{s} \otimes \phi^{-1}\right)$ is independent of $s$.

This tells us that $\operatorname{det} \bar{\rho}_{1}=\operatorname{det} \operatorname{Prim}[l]_{v, t} \otimes \phi^{-1}$ and $\operatorname{det} \bar{\rho}_{2}=\operatorname{det} \operatorname{Prim}\left[l^{\prime}\right]_{v, t^{\prime \prime}} \otimes \phi^{-1}$, and we can choose an isomorphism $\eta: \operatorname{det}\left(\operatorname{Prim}[M] \otimes \phi^{-1}\right) \rightarrow \operatorname{det} \bar{\rho}_{\mathbb{Z} / M \mathbb{Z}} \cdot$

In item (1) in the proof of Proposition 3.4.1, the set of automorphisms preserving this fixed isomorphism between determinants is seen to be $\operatorname{SL}(\mathbb{Z} / M \mathbb{Z})$. We know that the monodromy would be dense in this set, since $l, l^{\prime}>D$.

Finally, in item (3) in the proof, we see that the $\Omega_{\mathfrak{w}}$ are nonempty sets, since those above $l$ contain points above $t$ and those above $l^{\prime}$ contain points above $t^{\prime \prime}$.

It follows that there is a CM field $F_{1} / \mathbb{Q}\left(\mu_{10}\right)$ and a $t^{\prime} \in T_{\mathscr{W}}\left(F_{1}\right)$ satisfying the conditions (5)-(8) above. (For (6), namely Prim $\left.\left[l^{\prime}\right]_{v, t^{\prime}}\right|_{I_{F_{\mathfrak{w}}}} \equiv \epsilon_{l}^{-1} \oplus \epsilon_{l}^{-2}$, use the fact that Prim $\left[l^{\prime}\right]_{v, t^{\prime}}$ agrees with Prim $\left[l^{\prime}\right]_{t^{\prime \prime}}$, which was chosen to have this property.)

\footnotetext{
${ }^{2}$ We depart from the notation there in not writing $(\vec{h})$ for the twist by this character, since in fact $h(\sigma)=1$ for all $\sigma$, so one might think that $(\vec{h})$ means (1), but this is not true: $\phi^{-1}$ is not the cyclotomic character.
} 
Step 3. We will now apply Theorem 1.1.3 of [Barnet-Lamb 2008] to show that there exists a CM field $F / F_{1} / \mathbb{Q}\left(\mu_{10}\right)$ such that $\left.\operatorname{Prim}_{l^{\prime}, v, t^{\prime}} \otimes \phi^{-1}\right|_{\mathrm{Gal}(\overline{\mathbb{Q}} / F)}$ is automorphic, by taking $\mathscr{L}=\varnothing, N=10$, and $r=\operatorname{Prim}_{l, v, t^{\prime}} \otimes \phi^{-1}$. Let us verify the conditions of that theorem in turn. First of all, $l$ splits in $\mathbb{Q}\left(\mu_{10}\right)$ by our choice of $l$, and $q \nmid 10$ by our by choice of $q$. Then we address the numbered conditions:

(1) $r$ ramifies only at finitely many primes. This is trivial, being true for all Galois representations that come from geometry.

(2) $r^{c} \cong r^{\vee} \epsilon_{l}^{-1}$. For the same reason as for (3) (page 846), we have

$$
\operatorname{Prim}_{l, v, t^{\prime}}^{c}=\operatorname{Prim}_{l, v, t^{\prime}}^{\vee} \epsilon_{l}^{-3}
$$

whence we have what we want, since $r=\operatorname{Prim}_{l, v, t^{\prime}}^{\vee} \otimes \phi^{-1}$ and $\phi \phi^{c}=\epsilon_{l}^{-2}$.

(3) The Bellaïche-Chenevier sign is +1 . This is because the Poincaré duality pairing is symplectic and the multiplier of complex conjugation is odd.

(4) $r$ is crystalline with the right Hodge-Tate numbers. This follows immediately from the calculations of the previous proposition, once we note that the twist by $\phi$ changes the Hodge-Tate numbers by 1 .

(5) $r$ is unramified at all the primes of $\mathscr{L}$. This is vacuous.

(6) $\left.r\right|_{\operatorname{Gal}\left(\bar{F}_{v_{q}} / F_{v_{q}}\right)}$ ss is unramified and $\left.r\right|_{\operatorname{Gal}\left(\bar{F}_{v_{q}} / F_{v_{q}}\right)}$ ss has Frobenius eigenvalues 1, $\# k\left(v_{q}\right), \ldots,\left(\# k\left(v_{q}\right)\right)^{n-1}$. By [Katz 2009, Lemma 10.1] the local monodromy of Prim $\operatorname{liv,t}_{l, t^{\prime}} \otimes \overline{\mathbb{Q}}_{l}$ at $\infty$ is unipotent with a single Jordan block. We immediately deduce the same for $\operatorname{Prim}_{l, v, t^{\prime}}$. By the argument used to establish [Harris et al. 2010, Lemma 1.15], and recalling that $v_{q}\left(t^{\prime}\right)<0$, we conclude that inertia at $q$ acts via a maximal unipotent and that the Frobenius eigenvalues are of the form required.

(7) $\operatorname{det} r \equiv \epsilon_{l}^{-1}$. We saw above that $\operatorname{det}\left(\operatorname{Prim}[l]_{s} \otimes \phi^{-1}\right)=\epsilon_{l}^{-1}$, as required.

(8) Let $\bar{r}$ denote the semisimplification of the reduction of $r$, and let $r^{\prime}$ denote the extension of $r$ to a continuous homomorphism $\mathrm{Gal}\left(\bar{F} / F^{+}\right) \rightarrow \varphi_{n}\left(\overline{\mathbb{Q}}_{l}\right)$ (as described in [Clozel et al. 2008, §1]); then $\bar{r}^{\prime}\left(\operatorname{Gal}\left(\bar{F} / F\left(\zeta_{l}\right)\right)\right.$ is big (in the sense of "big image"). This is true by [Clozel et al. 2008, Lemma 2.5.5], since we chose $t^{\prime}$ such that Prim $\left[l^{\prime}\right]_{t^{\prime}} \equiv \operatorname{Prim}\left[l^{\prime}\right]_{t^{\prime \prime}}$, and we chose $t^{\prime \prime}$ such that $\operatorname{Gal}\left(\overline{\mathbb{Q}} / \mathbb{Q}\left(\mu_{10}\right)^{+}\right) \rightarrow \mathrm{GL}\left(\operatorname{Prim}\left[l^{\prime}\right]_{t^{\prime \prime}}\right)$ is surjective.

(9) $\bar{F}^{\text {ker ad } \bar{r}}$ does not contain $F\left(\zeta_{l}\right)$. This is true by the simplicity of $\operatorname{PSL}_{2}\left(\mathbb{F}_{l}\right)$ for $l>3$, again using the fact that $\operatorname{Gal}\left(\overline{\mathbb{Q}} / \mathbb{Q}\left(\mu_{10}\right)^{+}\right) \rightarrow \operatorname{GL}\left(\operatorname{Prim}\left[l^{\prime}\right]_{t^{\prime}}\right)$ is surjective.

(10) $r$ has the right restriction to inertia. This was guaranteed by the choice of $t^{\prime}$, once we note that the twist by $\phi$ changes restriction to inertia by $\epsilon_{l}$. 
(11) We can choose a polarization with a square determinant. This follows from the fact that $l^{\prime}$ splits in $F^{*}(v)$.

Having got that $\left.\operatorname{Prim}_{l^{\prime}, v, t^{\prime}} \otimes \phi^{-1}\right|_{\mathrm{Gal}(\overline{\mathrm{Q}} / F)}$ is also automorphic, we deduce that $\left.\operatorname{Prim}_{l, v, t^{\prime}} \otimes \phi^{-1}\right|_{\mathrm{Gal}(\overline{\mathbb{Q}} / F)}$ is automorphic since $Y_{t^{\prime}}$ has good reduction at $l$, since we chose $t^{\prime}$ such that $v_{\mathfrak{w}}\left(\left(t^{\prime}\right)^{5}-1\right)=0$ for w over $l$. Hence also $\operatorname{Prim} l, v,\left.t^{\prime}\right|_{\mathrm{Gal}(\overline{\mathbb{Q}} / F)}$ is automorphic.

Step 4. Next, I claim that $\left.\operatorname{Prim}_{l, v, t}\right|_{\mathrm{Gal}(\overline{\mathbb{Q}} / F)}$ is automorphic, by an appeal to Theorem 4.3.4 of [Clozel et al. 2008]. Conditions (1), (3), and (4) of that theorem are verified just like the corresponding conditions of [Barnet-Lamb 2008, Theorem 1.1.3], while (2) is trivial. We justify the remaining four conditions of Theorem 4.3.4 of [Clozel et al. 2008]:

(5) $r$ is discrete series somewhere. We saw in Step 2 that inertia at $q$ acts via a maximal unipotent, which suffices.

(6) $\bar{F}^{\text {ker ad } \bar{r}}$ does not contain $F\left(\zeta_{l}\right)$. True by the remarks just before Step 2.

(7) $\bar{r}^{\prime}\left(\operatorname{Gal}\left(\bar{F} / F\left(\zeta_{l}\right)\right)\right.$ is big. Again, true by the remarks just before Step 2.

(8) The residual representation is automorphic. Indeed, we have just verified that $\operatorname{Prim}[l]_{v, t^{\prime}}$ is automorphic, and Prim $[l]_{v, t^{\prime}} \equiv \operatorname{Prim}[l]_{v, t}$.

Step 5. We now use a rather standard argument to deduce the functional equation of the $L$ function from the potential automorphy which we have just derived. As a virtual representation of $\operatorname{Gal}(F / \mathbb{Q})$, we use Brauer's theorem to write

$$
1=\sum_{j} a_{j} \operatorname{Ind}_{\mathrm{Gal}\left(F / F_{j}\right)}^{\mathrm{Gal} F / \mathbb{Q}} \chi_{j},
$$

where the $F_{j}$ are intermediate fields between $F$ and $\mathbb{Q}$ with $\operatorname{Gal}\left(F / F_{j}\right)$ soluble, $a_{j} \in \mathbb{Z}$, and for each $j, \chi_{j}: \operatorname{Gal}\left(F / F_{j}\right) \rightarrow \mathbb{C}^{\times}$is an isomorphism. By solvable base change, since $\left.\operatorname{Prim}_{l, v, t}\right|_{\mathrm{Gal}(\overline{\mathbb{Q}} / F)}$ is automorphic, so is $\left.\operatorname{Prim}_{l, v, t}\right|_{\mathrm{Gal}\left(\overline{\mathbb{Q}} / F_{j}\right)}$ for each $j$; that is, we can find a RAESDC representation $\pi_{j}$ of weight 0 and type $\left\{\operatorname{Sp}_{n}(1)\right\}_{\{v \mid q\}}$ such that for any rational prime $l^{*}$ and isomorphism $\iota: \overline{\mathbb{Q}}_{l} \stackrel{\sim}{\rightarrow} \mathbb{C}$ we have

$$
\left.r_{l^{*}, l}\left(\pi_{j}\right) \equiv \operatorname{Prim}_{l^{*}, v, t}\right|_{\mathrm{Gal}\left(\overline{\mathbb{Q}} / F_{j}\right)},
$$

where

$$
\operatorname{Prim}_{l^{*}, v, t}=\sum_{j} a_{j} \operatorname{Ind}_{\mathrm{Gal}\left(F / F_{j}\right)}^{\mathrm{Gal} F / \mathbb{Q}} r_{l^{*}, \iota}\left(\pi_{j} \otimes\left(\chi_{j} \circ \operatorname{Art}_{F_{j}}\right)\right) .
$$

We deduce, using [Taylor and Yoshida 2007, Theorem 3.2 and Lemma 1.3(2)], that the $L$ function of $\operatorname{Prim}_{*, v, t}$ is defined and that

$$
L\left(\operatorname{Prim}_{*, v, t}\right)=\prod_{j} L\left(\pi_{j} \otimes\left(\chi_{j} \circ \operatorname{Art}_{F_{j}}\right), s\right)^{a_{j}},
$$


which gives the result, since each of the multiplicands on the right hand side obeys the expected functional equation, whence the left hand side does too.

We now put together what we know, to control the overall $L$ function of $\operatorname{Prim}_{l}$.

Corollary 6. The L function of $\operatorname{Prim}_{l}$ has meromorphic continuation to the whole complex plane, for $t \in \mathbb{Q}-\mathbb{Z}[1 / 10]$.

Proof. Proposition 2 gives us a list of pieces whose $L$ functions we must control. We may control Prim $l,[(0,0,0,0,0)]$ by Theorem 1 and the rest by Proposition 5 .

As we have set things up, the sheaf $\operatorname{Prim}_{l}$ has base defined over $\mathbb{Q}\left(\mu_{5}\right)$; but it could also have been defined over $\mathbb{Q}$ (unlike the various pieces $\operatorname{Prim}_{l, v}$, most of which are not defined over $\mathbb{Q}$, as $\operatorname{Gal}\left(\mathbb{Q}\left(\mu_{5}\right) / \mathbb{Q}\right)$ intermixes the various pieces). From now on, we will consider $\operatorname{Prim}_{l}$ to have been defined over $\mathbb{Q}$. Recapitulating the last part of the proof of Proposition 5 we get:

Theorem 7. The L function of $\operatorname{Prim}_{*, t}$ (now considered to be defined over $\mathbb{Q}$ ) has meromorphic continuation to the whole complex plane, for $t \in \mathbb{Q}-\mathbb{Z}[1 / 10]$.

Proof. By Steps 1-4 of the proof of Proposition 5, there are fields $F^{(v)}$ such that $\left.\operatorname{Prim}_{l, v, t}\right|_{\mathrm{Gal}\left(\overline{\mathbb{Q}} / F^{(v)}\right)}$ is automorphic for each $v$ in the table in Proposition 3; by the proof of Theorem 1 given in [Harris et al. 2010] the same is true for $v=(0,0,0,0,0)$, and by Proposition 2 , the symmetry of the situation allows us to deduce this for all other $v$. We can modify the proofs of these theorems to ensure that a single field extension $F$ makes all of these representations automorphic simultaneously. (For instance, the proof of [Harris et al. 2010, Theorem 3.1] can handle multiple representations simultaneously; there are no essential difficulties other than those of bookkeeping.) Then the whole sheaf Prim $\operatorname{lit}_{l, t}$ becomes automorphic when restricted to $G_{F}$.

We can then use the argument of Step 5 of the proof of Proposition 5 with $\operatorname{Prim}_{*, t}$ taking the place of $\operatorname{Prim}_{*, v, t}$ to deduce the expected functional equation for $L\left(\operatorname{Prim}_{*, t}, s\right)$ and thus meromorphic continuation.

Corollary 8. The zeta function of $Y_{t}$, for $t \in \mathbb{Q}-\mathbb{Z}[1 / 10]$, has meromorphic continuation.

Proof. By the remarks preceding Theorem 1, the remaining parts of the cohomology are well understood using the hard Lefschetz theorem.

\section{Concluding remarks}

We have seen that the zeta function of the hypersurface with projective equation

$$
\left(X_{1}^{5}+X_{2}^{5}+X_{3}^{5}+X_{4}^{5}+X_{5}^{5}\right)=5 t X_{1} X_{2} \ldots X_{5}
$$

has a meromorphic continuation and satisfies the expected functional equation. 
It is perhaps natural to wonder whether the techniques used might generalize to more general hypersurfaces of a similar type. For instance, [Harris et al. 2010] shows that the $\Gamma_{W} / \Delta$ invariants in the cohomology of the variety

$$
\left(X_{1}^{N}+X_{2}^{N}+X_{3}^{N}+X_{4}^{N}+\cdots+X_{N}^{N}\right)=N t X_{1} X_{2} \ldots X_{N}
$$

will be automorphic for all odd $N$, so we might wonder whether the result of this paper can be generalized to other $N$ 's. The methods of [Katz 2009] work in an even more general context, replacing the monomial $X_{1} X_{2} \ldots X_{N}$ on the right-hand side of the defining equation with an arbitrary monomial of the required degree, so one might also ask if there are any cases of that form to which we might try to generalize the result of this paper. I feel that a few remarks on these cases may be useful to the reader.

5.1. Smaller $N$ 's in (9). It is worth beginning by noting that the cases $N=1,2$ are trivial, and the case $N=3$ is also uninteresting since then (9) describes a family of elliptic curves, and the zeta function is already understood. Thus the only interesting case with smaller $N$ is $N=4$.

At first sight, it might seem difficult to analyze this case using the methods of this paper, since the result of [Harris et al. 2010] which gives the automorphicity of $\operatorname{Prim}_{l,[(0, \ldots, 0)]}$ requires $N$ to be odd. But [Barnet-Lamb 2010] generalizes their methods to cover odd-dimensional cases, and it is then possible to extend the methods of this paper to cover that case, too. In particular, an analysis like that in Section 3 will reveal that all the pieces of the cohomology apart from Prim $l,[(0,0,0,0)]$ are one- or zero-dimensional, and so trivially automorphic.

I have chosen not to give this argument in full detail, since a very beautiful geometric argument of N. Elkies and M. Schütt (personal communication) tells us that, for the case $N=4$, each Dwork hypersurface is isogenous to the Kummer surface of a product $E_{1} \times E_{2}$, where $E_{1}$ and $E_{2}$ are elliptic curves defined over a quadratic extension of $\mathbb{Q}$, conjugate to each other over $\mathbb{Q}$, and related by a 2isogeny. This allows one to quite directly see the automorphicity required in this case, and little would be served by giving the full details of the argument above.

5.2. Larger values of $N$ in (9). If we try to extend the methods of this paper to larger $N$, we face the following problem.

Proposition 9. (1) Let $N \geq 8$ be an integer. Then the Hodge-Tate numbers of $\operatorname{Prim}_{l,[(4, N-2, N-2,0, \ldots, 0)]}$ include 2 with multiplicity at least 2.

(2) Let $N=6$. Then the Hodge-Tate numbers of $\operatorname{Prim}_{l,[(0,0,0,2,2,2)]}$ include 3 with multiplicity at least 2 .

Proof. Again, we use [Katz 2009, Lemma 3.1]. For the first statement, the totally nonzero representatives include $(5, N-1, N-1,1, \ldots, 1)$ and $(7,1,1,3, \ldots, 3)$. 
We have

$$
\frac{5+(N-1)+(N-1)+1+\cdots+1}{N}-1=\frac{7+1+1+3+\cdots+3}{N}-1=2,
$$

so 2 occurs as a Hodge-Tate weight with multiplicity at least 2 .

The proof of the second statement is similar: consider the nonzero representatives $(2,2,2,4,4,4)$ and $(5,5,5,1,1,1)$.

Thus in all cases with $N$ even (recall that we need $N$ even for [Harris et al. 2010] to apply ${ }^{3}$ ) and $N \geq 6$, at least one of the pieces of the cohomology of (9) will have a repeated Hodge-Tate number. At present, apart from some work in the case of two-dimensional Galois representations, there are no modularity lifting theorems for representations with repeated Hodge-Tate numbers, and hence (since such theorems are a key ingredient in proving the potential modularity theorems such as [Taylor 2006; Harris et al. 2010] on which this paper relies) it seems unlikely that the approach of this paper can be extended to cover such cases.

(One might briefly wonder whether some larger algebra of correspondences could be used to cut the cohomology into smaller pieces, small enough that they no longer have repeated Hodge-Tate weights; but this is impossible, since the results of Katz on the monodromy of the cohomology tell us that all the pieces in the decomposition of the cohomology into eigenspaces for $\Gamma_{W} / \Delta$ cannot be broken up further, as the monodromy acts transitively on each piece.)

5.3. Other monomials in (9). Katz studies the more general equation

$$
\left(X_{1}^{N}+X_{2}^{N}+X_{3}^{N}+\cdots+X_{N}^{N}\right)=N \lambda \prod_{i} X_{i}^{w_{i}},
$$

where $W=\left(w_{1}, \ldots, w_{N}\right)$ is a sequence of nonnegative integers summing to $N$. It is natural to ask whether the methods of this paper can be extended to any varieties of this form, beyond the cases already considered. Unfortunately, the answer is no.

Let's imagine an analysis based on the same techniques used above. As before, the main challenge would be to analyze the middle-dimensional cohomology, since the rest is determined by hard Lefschetz. We can define $\operatorname{Prim}_{l}^{N-2}$, as in [Katz 2009], to correspond to the part of the middle-dimensional cohomology not coming from Lefschetz. Following the method above, our next step is to decompose this cohomology into eigensheaves.

The natural group acting on (10) is easily seen to be

$$
\left\{\left(\zeta_{1}, \ldots, \zeta_{N}\right) \in\left(\mu_{N}\right)^{N} \mid \prod \zeta_{i}^{w_{i}}=1\right\} / \Delta,
$$

where $\Delta$, as before, is $\mu_{N}$ embedded diagonally. The character group in this case is

\footnotetext{
${ }^{3}$ Even if this were not an obstacle, the $N=7$ case also has a piece of the cohomology with a repeated Hodge-Tate number.
} 
$(\mathbb{Z} / N \mathbb{Z})_{0} /\langle W\rangle$, where we abuse notation by considering $W$ as a class in $(\mathbb{Z} / N \mathbb{Z})_{0}$. We will write an element of $(\mathbb{Z} / N \mathbb{Z})_{0} /\langle W\rangle$ as either $v \bmod W$ or simply $[v]$ and define $\operatorname{Prim}_{l,[v]}^{N-2}$ in a similar manner as before.

Suppose now that we have fixed some $W$. The main challenge in applying the methods to this paper to show that the zeta function of the family (10) is meromorphic will be showing that $\operatorname{Prim}_{l,[v]}^{N-2}$ is automorphic for each $v$. Since this will rely, in the final analysis, on the application of a lifting theorem, we will certainly require that $\operatorname{Prim}_{l,[v]}^{N-2}$ has distinct Hodge numbers for all $v$. This, unfortunately, will never happen except in the cases already considered.

Proposition 10. Suppose $N \geq 3$ and $W \neq(1,1, \ldots, 1)$. Then there exists some $v$ such that the Hodge-Tate numbers of $\operatorname{Prim}_{l,[v]}^{N-2}$ are not all distinct.

Proof. Since $W \neq(1,1, \ldots, 1)$, some $w_{i}$ - say $w_{1}$ - vanishes. For $i>1$, write $h_{i}$ for $\operatorname{hcf}\left(w_{i}, N\right)$. For each $i>1$ where $h_{i}=1$, set $v_{i}=0$; then, for $k \in \mathbb{Z}$,

$$
v_{i}+k w_{i} \equiv 0(\bmod N) \quad \Longleftrightarrow \quad k \equiv 0(\bmod N) .
$$

For $i>1$ where $h_{i}>1$, we can choose $v_{i}=1$; then $v_{i}+k w_{i}$ will always be $\equiv 1 \bmod h_{i}$, and we will never have $v_{i}+k w_{i} \equiv 0 \bmod N$. Finally, $v_{1}$ is fixed by the condition that the $v_{i}$ sum to 0 . (Note that $\tilde{v}_{1}>1$, since if not $h_{i}=1$ for all $i>1$, hence $v_{i}>1$ for all such $i$, which is impossible.)

The elements of $(\mathbb{Z} / N \mathbb{Z})_{0}$ representing $[v]$ are $v+k W$ for $k \in \mathbb{Z} / N \mathbb{Z}$; it is immediate that the element $v+k W$ is totally nonzero for all $k \in \mathbb{Z} / N \mathbb{Z}$ except $k=0$, so the totally nonzero representatives are $\{v, v+W, \ldots, v+(N-1) W\}$, and the multiset of Hodge-Tate numbers is the multiset

$$
\{\operatorname{deg}(v)-1, \operatorname{deg}(v+W)-1, \operatorname{deg}(v+2 W)-1, \ldots, \operatorname{deg}(v+(N-1) W-1\} .
$$

If we suppose for contradiction that these numbers are distinct, the $N-1$ integers $\operatorname{deg}(v+W), \operatorname{deg}(v+2 W), \ldots, \operatorname{deg}(v+(N-1) W)$ are distinct. We now note that the degree of an element of $(\mathbb{Z} / N \mathbb{Z})_{0}$ is trivially $\leq N-1$, and that, writing $u$ for some $v+k W$, we have $N \operatorname{deg} u \geq \tilde{u}_{1}+(N-1)$ since $u$ is totally nonzero), and this in turns equals $\tilde{v}_{1}+(N-1)>N$, since $w_{1}=0$. Since $\operatorname{deg}(v+k W)>1$, we have $N-1$ distinct integers $\operatorname{deg}(v+k W)$ with $1<\operatorname{deg}(v+k W) \leq N-1$, a contradiction.

Acknowledgements. I thank my advisor, Richard Taylor, for suggesting this problem to me and for his help in pursuing it. I am grateful to Barry Mazur for helpful suggestions made concerning this material when he examined it as part of my $\mathrm{PhD}$ thesis. I also thank the anonymous referee for further helpful suggestions. 


\section{References}

[Barnet-Lamb 2008] T. Barnet-Lamb, "Potential automorphy for certain Galois representations to GL(2n)", preprint, 2008. To appear in J. Reine Angew. Math. arXiv 0811.1586v1

[Barnet-Lamb 2010] T. Barnet-Lamb, "On the potential automorphy of certain odd-dimensional Galois representations”, Compos. Math. 146:3 (2010), 607-620. MR 2644929 Zbl 05722159

[Barnet-Lamb et al. 2010] T. Barnet-Lamb, T. Gee, D. Geraghty, and R. Taylor, "Potential automorphy and change of weight", preprint, 2010. arXiv 1010.2561v1

[Clozel et al. 2008] L. Clozel, M. Harris, and R. Taylor, "Automorphy for some $l$-adic lifts of automorphic mod $l$ Galois representations", Publ. Math. Inst. Hautes Études Sci. 108 (2008), 1-181. MR 2470687 (2010j:11082) Zbl 1169.11020

[Deligne et al. 1982] P. Deligne, J. S. Milne, A. Ogus, and K.-Y. Shih, Hodge cycles, motives, and Shimura varieties, Lecture Notes in Mathematics 900, Springer, Berlin, 1982. MR 84m:14046 Zbl 0465.00010

[Ekedahl 1990] T. Ekedahl, “An effective version of Hilbert's irreducibility theorem”, pp. 241-249 in Séminaire de Théorie des Nombres (Paris, 1988-1989), edited by C. Goldstein, Progr. Math. 91, Birkhäuser, Boston, MA, 1990. MR 92f:14018 Zbl 0729.12005

[Harris et al. 2010] M. Harris, N. Shepherd-Barron, and R. Taylor, "A family of Calabi-Yau varieties and potential automorphy", Ann. of Math. (2) 171:2 (2010), 779-813. MR 2630056 Zbl 05712744

[Katz 1988] N. M. Katz, Gauss sums, Kloosterman sums, and monodromy groups, Annals of Mathematics Studies 116, Princeton University Press, 1988. MR 91a:11028 Zbl 0675.14004

[Katz 2009] N. M. Katz, "Another look at the Dwork family", pp. 89-126 in Algebra, arithmetic, and geometry: in honor of Y. I. Manin, vol. II, edited by Y. Tschinkel, Progr. Math. 270, Birkhäuser, Boston, 2009. MR 2641188 Zbl 1195.14015

[Larsen 1995] M. Larsen, "Maximality of Galois actions for compatible systems", Duke Math. J. 80:3 (1995), 601-630. MR 97a:11090 Zbl 0912.11026

[Matthews et al. 1984] C. R. Matthews, L. N. Vaserstein, and B. Weisfeiler, "Congruence properties of Zariski-dense subgroups, I”, Proc. London Math. Soc. (3) 48:3 (1984), 514-532. MR 85d:20040 Zbl 0551.20029

[Nori 1987] M. V. Nori, "On subgroups of $\mathrm{GL}_{n}\left(\mathbf{F}_{p}\right)$ ", Invent. Math. 88:2 (1987), 257-275. MR 88d:20068 Zbl 0632.20030

[Scholl 2006] A. J. Scholl, "On some $l$-adic representations of Gal $(\overline{\mathbb{Q}} / \mathbb{Q})$ attached to noncongruence subgroups”, Bull. London Math. Soc. 38:4 (2006), 561-567. MR 2007c:11065 Zbl 1126.11029

[Taylor 2006] R. Taylor, "On the meromorphic continuation of degree two L-functions", Doc. Math. Extra Vol. (2006), 729-779. MR 2008c:11154 Zbl 1138.11051

[Taylor and Yoshida 2007] R. Taylor and T. Yoshida, "Compatibility of local and global Langlands correspondences", J. Amer. Math. Soc. 20:2 (2007), 467-493. MR 2007k:11193 Zbl 05120650

Communicated by Hélène Esnault

Received 2009-01-16 Revised 2010-08-09 Accepted 2010-08-10

tbl@math.harvard.edu

Department of Mathematics, Brandeis University, 415 South Street MS 050, Waltham, MA 02453, United States http://people.brandeis.edu/ ${ }^{\text {tbl/ }}$ 


\section{Algebra \& Number Theory}

www.jant.org

\section{EDITORS}

\section{MANAGING EDITOR}

Bjorn Poonen

Massachusetts Institute of Technology

Cambridge, USA

\author{
EDITORIAL BOARD CHAIR \\ David Eisenbud \\ University of California \\ Berkeley, USA
}

\section{BOARD OF EDITORS}

\section{Georgia Benkart}

Dave Benson

Richard E. Borcherds

John H. Coates

J-L. Colliot-Thélène

Brian D. Conrad

Hélène Esnault

Hubert Flenner

Edward Frenkel

Andrew Granville

Joseph Gubeladze

Ehud Hrushovski

Craig Huneke

Mikhail Kapranov

Yujiro Kawamata

János Kollár

Hendrik W. Lenstra

Yuri Manin

Barry Mazur
University of Wisconsin, Madison, USA

University of Aberdeen, Scotland

University of California, Berkeley, USA

University of Cambridge, UK

CNRS, Université Paris-Sud, France

University of Michigan, USA

Universität Duisburg-Essen, Germany

Ruhr-Universität, Germany

University of California, Berkeley, USA

Université de Montréal, Canada

San Francisco State University, USA

Hebrew University, Israel

University of Kansas, USA

Yale University, USA

University of Tokyo, Japan

Princeton University, USA

Universiteit Leiden, The Netherlands

Northwestern University, USA

Harvard University, USA
Susan Montgomery

Shigefumi Mori

Andrei Okounkov

Raman Parimala

Victor Reiner

Karl Rubin

Peter Sarnak

Michael Singer

Ronald Solomon

Vasudevan Srinivas

J. Toby Stafford

Bernd Sturmfels

Richard Taylor

Ravi Vakil

Michel van den Bergh

Marie-France Vignéras

Kei-Ichi Watanabe

Andrei Zelevinsky

Efim Zelmanov
University of Southern California, USA

RIMS, Kyoto University, Japan

Princeton University, USA

Emory University, USA

University of Minnesota, USA

University of California, Irvine, USA

Princeton University, USA

North Carolina State University, USA

Ohio State University, USA

Tata Inst. of Fund. Research, India

University of Michigan, USA

University of California, Berkeley, USA

Harvard University, USA

Stanford University, USA

Hasselt University, Belgium

Université Paris VII, France

Nihon University, Japan

Northeastern University, USA

University of California, San Diego, USA

\section{PRODUCTION}

ant@mathscipub.org

Silvio Levy, Scientific Editor

Andrew Levy, Production Editor

See inside back cover or www.jant.org for submission instructions.

The subscription price for 2010 is US \$140/year for the electronic version, and \$200/year (+\$30 shipping outside the US) for print and electronic. Subscriptions, requests for back issues from the last three years and changes of subscribers address should be sent to Mathematical Sciences Publishers, Department of Mathematics, University of California, Berkeley, CA 94720-3840, USA.

Algebra \& Number Theory (ISSN 1937-0652) at Mathematical Sciences Publishers, Department of Mathematics, University of California, Berkeley, CA 94720-3840 is published continuously online. Periodical rate postage paid at Berkeley, CA 94704, and additional mailing offices.

ANT peer review and production are managed by EditFLOW ${ }^{\mathrm{TM}}$ from Mathematical Sciences Publishers.

\section{PUBLISHED BY}

mathematical sciences publishers

http://www.mathscipub.org

A NON-PROFIT CORPORATION

Typeset in LATEX

Copyright $\odot 2010$ by Mathematical Sciences Publishers 


\section{Algebra \& Number Theory}

Volume $4 \quad$ No. $7 \quad 2010$

Hochschild cohomology and homology of quantum complete intersections

STEFFEN OPPERMANN

Meromorphic continuation for the zeta function of a Dwork hypersurface

THOMAS BARNET-LAMB

Equations for Chow and Hilbert quotients

ANGEla Gibney and Diane MaClagan

Haglund-Haiman-Loehr type formulas for Hall-Littlewood polynomials of type $B$ and C

\section{CRISTIAN LENART}

On exponentials of exponential generating series

ROLAND BACHER

On families of $\varphi, \Gamma$-modules

KIRAN KEDLAYA and RUOCHUAN LIU 